\title{
Ultrafast Imaging at All Frequencies
}

\section{A new algorithm could allow researchers to capture attosecond, multiwavelength images of an object.}

\section{By Marric Stephens}

lluminating a sample with attosecond x-ray pulses could let researchers image phenomena as fleeting as the rearrangement of electrons during chemical reactions (see Synopsis: Jumpstarting Electron Motion in Molecules). The uncertainty principle dictates that ultrashort pulses have a broad energy spectrum. However, because focusing different wavelengths typically requires multiple sets of optics, most attempts at attosecond imaging are spectroscopic, ignoring all but one radiation frequency. Now, Jianwei Miao at the University of California, Los Angeles, and colleagues have developed an innovative algorithm that can simultaneously reconstruct multiple images of an object at different wavelengths using attosecond pulses [1]. The method offers a way to take spectroscopic images without the need for sophisticated instruments.

In simulations, the team scanned a micrometer-sized object with a series of broadband, attosecond x-ray pulses and collected the resulting diffraction patterns. They then fed these measurements into their algorithm, which used them in an

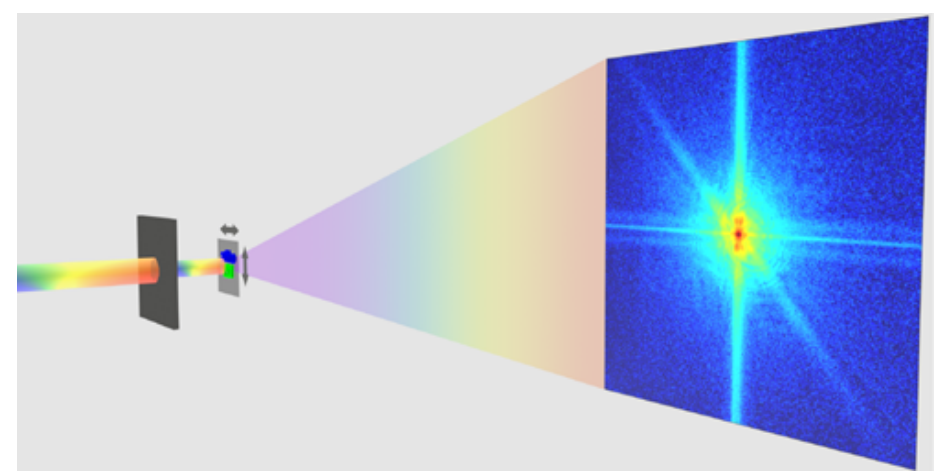

Credit: A. Rana et al., Phys. Rev. Lett. (2020) iterative process that reconstructed the incident beam and the object's properties for 17 individual $x$-ray wavelengths. The algorithm searched for the optimal fit among these 17 wavelengths and used it as a constraint to improve the other 16 . After a few hundred iterations, the algorithm obtained a reliable image for all 17 wavelengths.

To validate their approach, the team conducted an experiment using visible-light pulses from a broadband LED. They reconstructed 17 spectral images of a test object by measuring the diffraction patterns projected onto a CCD camera. Their result suggests that the technique can be applied to a variety of light sources, potentially allowing, for example, multiple chemical-specific images of materials and biological samples to be obtained simultaneously using broadband illumination.

Marric Stephens is a Corresponding Editor for Physics based in Bristol, UK.

\section{REFERENCES}

1. A. Rana et al., "Potential of attosecond coherent diffractive imaging," Phys. Rev. Lett. 125, 086101 (2020). 